\title{
Adriaan van Roomen y la uranografía: el enlace de las disciplinas matemáticas y de la filosofía en los siglos XVI y XVII*
}

\author{
Adriaan van Roomen and the Uranography: The Link between Mathematical \\ Disciplines and Philosophy in the 16th and 17th Centuries
}

Zaqueu Vieira Oliveira ${ }^{\dagger}$

\begin{abstract}
Resumen
Las obras astronómicas publicadas en el siglo XVI seguramente pueden ser organizadas según dos características: algunas abordan las cuestiones filosóficas acerca de los cuerpos celestes y otras tratan de los problemas técnicomatemáticos de la astronomía. Sin embargo, en el siglo siguiente, aunque las publicaciones continuaran abordando la astronomía de esos dos modos, surgió un tipo de obra que intentaba unir las características filosóficas y matemáticas de la astronomía. En este trabajo, nos fijaremos en la Ouranographia de Adriaan van Roomen (1561-1615), una de las primeras obras que unen el conocimiento filosófico y la tecnicidad de las matemáticas para fundamentar la astronomía -y también entraremos indirectamente en los debates acerca de la quaestio de certitudine mathematicarum-. Con eso, van Roomen no solamente publica una obra innovadora, sino que también -en medio a un intenso debate acerca de la clasificación del conocimiento y de las matemáticas- crea una "nueva" disciplina, la uranografía.
\end{abstract}

Palabras clave: astronomía - matemáticas - filosofía - quaestio de certitudine mathematicarum

\begin{abstract}
Astronomical works published in the sixteenth century can certainly be categorized according to two characteristics: some works dealing with the philosophical questions related to celestial bodies and others dealing with technical/mathematical problems of astronomy. In the following century, although astronomical publications continued to approach subject matter in the aforementioned ways, it seems to have emerged a type of work that tried to unite the philosophical and mathematical aspects of astronomy. In this paper, we will approach the Ouranographia of Adriaan van Roomen (1561-1615), one of the first works to ground astronomy on a merger of philosophical knowledge and the technical aspects of mathematics - indirectly entering discussions about the 'quaestio de certitudine mathematicarum'. Thus, van Roomen not only published a groundbreaking work, but also - in the midst of an intense debate about the classification of knowledge and mathematics - created a "new" discipline, uranography.
\end{abstract}

Keywords: astronomy - mathematics - philosophy - quaestio de certitudine mathematicarum

\footnotetext{
* Recibido: 22 de octubre de 2017. Aceptado con revisiones: 9 de febrero de 2018.

† Universidade de São Paulo, Brasil. Para contactar al autor, por favor, escribir a: z.zaqueu@usp.br. Metatheoria 9(1)(2018): 13-27. ISSN 1853-2322. eISSN 1853-2330.

(c) Editorial de la Universidad Nacional de Tres de Febrero. Publicado en la República Argentina.
} 


\section{Introducción}

El Adriaan van Roomen, el personaje principal de este artículo, nació el 29 de septiembre de 1561 en la ciudad de Leuven (actual Bélgica) y murió el 4 de mayo de 1615 en Mainz (Alemania) retornando de un viaje que hacía por cuestiones de salud (Busard 1981).

No existen informaciones sobre la infancia y juventud de van Roomen. Sabemos que estudió matemáticas y filosofía en el Colegio de los Jesuitas de Colonia (Alemania) y, después, estudió medicina primero en Colonia, luego en la Universidad de Leuven y en la Universidad de Bolonia (Italia). Su principal actividad fue la enseñanza de la medicina y de las matemáticas. Entre 1586 y 1592 , fue profesor de matemáticas y de medicina en la Universidad de Leuven y luego profesor de medicina en la Universidad de Würzburg entre 1593 y 1607 . También tuvo ocupaciones en la catedral y en la iglesia de Neumünster en Würzburg y también en la iglesia de Leuven, pero sus viajes y problemas de salud lo han impedido de realizar sus actividades religiosas como le gustaría. Años después, entre 1610 y 1612, fue profesor de matemáticas de Thomas Zamojski, hijo del creador de la escuela de Zamosc (Polonia), Jan Zamojski (Busard 1981).

Van Roomen, aunque no fuera un matemático y astrónomo que hoy consideramos protagonista de la ciencia de su tiempo, fue un autor relevante y original. Podemos decir que fue un típico experto de su tiempo, porque participaba asiduamente de las ferias del libro de Mainz y Frankfurt y buscaba las publicaciones más recientes de sus áreas de interés: matemáticas, astronomía, medicina, filosofía, etc. Las ferias también representaban un momento de enviar o recibir cartas y libros para/de otros estudiosos a través de los libreros.

El estudioso belga mantuvo contacto a través de correspondencia con muchos expertos contemporáneos, como los padres jesuitas Christoph Clavius (1538-1612) y Christoph Grienberger (1561-1636), los astrónomos Johannes Kepler (1571-1630) y Tycho Brahe (1546-1601) y los matemáticos François Viète (1540-1603) y Ludolph van Ceulen (1540-1610). Según Bockstaele (1976, 1992) actualmente existen 47 cartas, dado que 19 de ellas fueron enviadas por van Roomen a Clavius. Entre las demás, se incluyen cartas intercambiadas con el astrónomo Giovanni Antonio Magini (15551617), el impresor, librero y editor Jan Moretus (1543-1610), el patrono de Kepler, Herwart von Hohenburg (1553-1622), el matemático Jan Brozek (1585-1652), el humanista Joseph Justus Scaliger (1540-1609) y otros. Desafortunadamente, las cartas intercambiadas con Viète, Brahe y van Ceulen se han perdido (Bockstaele 1976, pp. 87-90, Bockstaele 1992, pp. 1-3).

La mayoría de las obras de van Roomen tienen como tema principal la medicina (aunque debamos tener en cuenta que esos trabajos son tesis escritas por sus alumnos en las universidades que trabajaba) (Ruland 1867), pero su originalidad está en las matemáticas. Sus obras matemáticas tienen enfoque principal en la geometría y trigonometría. Cuando van Roomen es recordado en la historia de la matemática comúnmente aparece en las páginas sobre el número $\pi$ que él publicó el valor con 16 cifras decimales en la obra Ideae mathematicae pars prima (van Roomen 1593). El matemático belga también es personaje importante en el desarrollo de la mathesis universalis -la búsqueda de un conocimiento general que pudiera funcionar como base para todas las ciencias- con posibles influencias en la obra cartesiana Reglas para la dirección del espíritu (Oliveira 2015, Sasaki 2004, Weber 1964).

Van Roomen también publicó en 1591 una obra astronómica que creemos ser de gran importancia para la historia de la astronomía y de la ciencia en los siglos XVI y XVII, la Ouranographia sive caeli descriptio (van Roomen 1591). En este artículo enfatizaremos la originalidad y la importancia del pensamiento de van Roomen en su descripción de la uranografía, disciplina que, a juicio del autor, está entre las matemáticas y la filosofía. Estudiar la relación entre matemáticas y filosofía en finales del siglo XVI también es importante para las discusiones más generales acerca de la historia de las disciplinas científicas, pues, como nos dice Horacio Capel:

a través de la historia de la disciplina se puede observar la posición que adopta un científico en las polémicas y en los cambios que afectan a su ciencia tanto por sus citaciones y por la valoración que 
realiza de los precedentes y antepasados, como por las omisiones y silencios, y, desde luego, por la misma selección de los materiales incluidos. (Capel 1989, s/p)

A lo largo de este artículo intentaremos aclarar cómo las posiciones adoptadas por van Roomen estaban conectadas a los cambios y permanencias de las matemáticas de su tiempo, tanto del punto de vista de los debates internos de las matemáticas, como de las cuestiones sociales relacionadas.

En las tres secciones siguientes, intentaremos mostrarle al lector algunos temas ya conocidos de la historia de las ciencias en los siglos XVI y XVII, pero son el fondo de las discusiones en las secciones finales del artículo. Abordaremos la quaestio de ceritudine mathematicarum (sección 2), la clasificación de las matemáticas (sección 3) y las categorías de obras astronómicas comúnmente publicadas en el siglo XVI y los cambios que ocurrieron en el siglo siguiente (sección 4). En la sección 5, describiremos la obra Ouranographia sive caeli descriptio (1591) de van Roomen. Concluiremos el artículo (sección 6) haciendo la defensa de que la uranografía de van Roomen hizo un enlace de las matemáticas con la filosofía para, además de asegurar que las matemáticas poseían conocimiento seguro, confirmar que también tenían raíz en la filosofía antigua y medieval.

\section{La quaestio de certitudine mathematicarum y van Roomen}

Durante los siglos XVI y XVII ocurrió un conocido debate entre los matemáticos y los filósofos acerca de la certeza del conocimiento producido por las disciplinas matemáticas, la quaestio de certitudine mathematicarum o "cuestión de la certeza de las matemáticas".

Según Luis Miguel Carolino (2006), en los siglos XVI y XVII, hubo una gran tensión entre matemáticos y filósofos en la Compañía de Jesús que afectaba el ámbito institucional e incluso el propio currículo jesuita: ellos colocaban las disciplinas matemáticas en una posición subalterna en relación a la filosofía natural, pero eso no era aceptado por los matemáticos de la Compañía. Los filósofos y matemáticos jesuitas estaban envueltos en un debate que intentaba probar si las matemáticas podrían o no ser consideradas ciencia según la lógica y la ontología aristotélica (Carolino 2006, pp.18). Esos debates no fueron restrictos a los colegios jesuitas, sino que también ocurrieron dentro del ambiente de muchas universidades europeas (Mancosu 1996).

A través de sus cartas, nos damos cuenta de que van Roomen estuvo directamente afectado por ese estatuto "inferior" de las matemáticas dentro de las universidades en las cuales trabajaba, de manera que tenía que dedicarse más a las actividades de enseñanza de la medicina y renunciar a los estudios matemáticos que le interesaban tanto. El tiempo escaso también afectaba las actividades y estudios de las disciplinas matemáticas de van Roomen. En una carta enviada a Clavius el 11 de noviembre de 1593, él enfatiza que las actividades como profesor de medicina ocupaban su tiempo: "En lo relacionado con mis estudios, mucho de la profesión médica retarda las matemáticas, porque estoy aquí solamente ejerciendo el cargo de profesor, si fuera de otro modo, yo tendría progresos más significativos en la tabla de senos; sin embargo, trabajo lentamente, brevemente con la ayuda de la gracia divina, voy a editar algún espécimen” (Bockstaele 1976, pp. 106).

Sin que entremos, todavía, directamente en el debate acerca del estatuto epistemológico de las disciplinas matemáticas que ocurría en aquel tiempo, queremos aquí demonstrar que la idea de uranografía de van Roomen podría tener la meta de crear un enlace entre las matemáticas y la filosofía. Tal vez, el autor buscaba demostrar que las matemáticas poseían un lugar tan importante como el ocupado por la filosofía en la clasificación del conocimiento.

Otra obra de gran importancia del autor es la In Archimedis circuli dimensionem expositio et análisis (1597). En esa obra, van Roomen define el concepto de mathesis universalis, una ciencia general que podría servir como base para demonstrar todas las demás disciplinas matemáticas. Esa ciencia, que tenía como punto de partida las ideas de la teoría de las proporciones de Eudoxo y el quinto libro de los Elementos (2009) de Euclides, proporcionaría a las matemáticas más certeza y precisión que la aritmética y la geometría (van Roomen 1597, pp. 23). Esa obra también puede estar inserida en los intentos de van Roomen de valorar las matemáticas frente al conocimiento filosófico. Según Giovani 
Crapulli (1969), van Roomen puede haber sido el matemático que describió la mathesis universalis más detalladamente y, además, Jean-Paul Weber (1964) y Chikara Sasaki (2004) nos presentan evidencias de que René Descartes se haya basado en van Roomen para la escritura de su obra Regulae ad directionem ingenii (1684).

\section{La clasificación de las matemáticas}

Es necesario resaltar que, durante toda la historia, la organización y clasificación del conocimiento fue diversa y compleja. En el período que nos interesa, los siglos XVI y XVII, las disciplinas matemáticas fueron consideradas por muchos estudiosos de manera muy diferente de la manera actual (Knobloch 1989). Van Roomen se dedicó intensamente a este asunto como veremos más adelante.

En el siglo XVI, las matemáticas fueron entendidas a partir de un conjunto de disciplinas que venía de una tradición más antigua influenciada por muchos autores de la antigüedad y de la edad media como Platón, Aristóteles, Anatolio de Alexandria, Proclo, Isidoro de Sevilla y también los más recientes, como Luca Pacioli- que escribieron en sus obras consideraciones acerca de las divisiones y partes que deberían formar ese grupo de disciplinas. El concepto más aceptado y común en aquel tiempo es exactamente las disciplinas matemáticas del quadrivium medieval: aritmética, música, geometría y astronomía (Gonçalves \& Oliveira 2010, Knobloch 1989).

Pero, debemos tener en cuenta que los expertos de los siglos XVI y XVII comprendían las matemáticas como un gran conjunto de disciplinas más amplio que el quadrivium. Tenemos que recordar que no había un consenso en esa clasificación, sino muchos trabajos que contenían distintas clasificaciones. Aquí, no voy a describir tales clasificaciones, pero podemos resaltar que muchas de esas organizaciones poseían por lo menos dos grupos de disciplinas: (i) las matemáticas puras (las disciplinas que abordan las cantidades abstractas) como la aritmética y la geometría y (ii) las matemáticas mixtas (las disciplinas que buscan el conocimiento de las cantidades concretas) como la astronomía, la música, la geografía y la óptica (Knobloch 1989).

Van Roomen publicó su clasificación de las matemáticas inicialmente en dos obras: Universae mathesis idea (1602) y Mathesis polemica (1605) que trata del uso de las disciplinas matemáticas en la actividad bélica.

La clasificación de van Roomen, así como otras publicadas en los siglos XVI y XVII, afirma que las matemáticas tienen como objeto de estudio, las cantidades, en general, consideradas como algo posible de ser medido (Abbagnano 2007, pp. 818). Pero, las definiciones más tradicionales solamente presentan los conocimientos aritméticos y geométricos. Van Roomen va más allá:

También, los matemáticos han considerado más allá de las cantidades, como algunos accidentes, por ejemplo, el movimiento, el peso, el sonido, el radio, etc., así como las substancias, el cielo, la tierra, los campos, los cerros, etc. igualmente las cosas artificiales, como barriles, esferas, etc. No los considera solamente como tales, pero también como cuantificables (van Roomen 1605, pp. 6).

Siguiendo esa idea, la clasificación de van Roomen posee cerca de veinte disciplinas, como es posible ver en el diagrama de la Mathesis polemica (1605, figura 1). Inicialmente, van Roomen hace una distinción entre las disciplinas matemáticas principales -aquellas que buscan comprender solamente las cantidades- y las mecánicas -disciplinas que comúnmente no están entre las matemáticas, pero el autor las considera porque de algún modo necesitan del conocimiento de las matemáticas principales-. Las matemáticas mecánicas son aquellas disciplinas que buscan comprender la producción, el uso y las acciones de los instrumentos mecánicos (van Roomen 1605, pp. 15). 


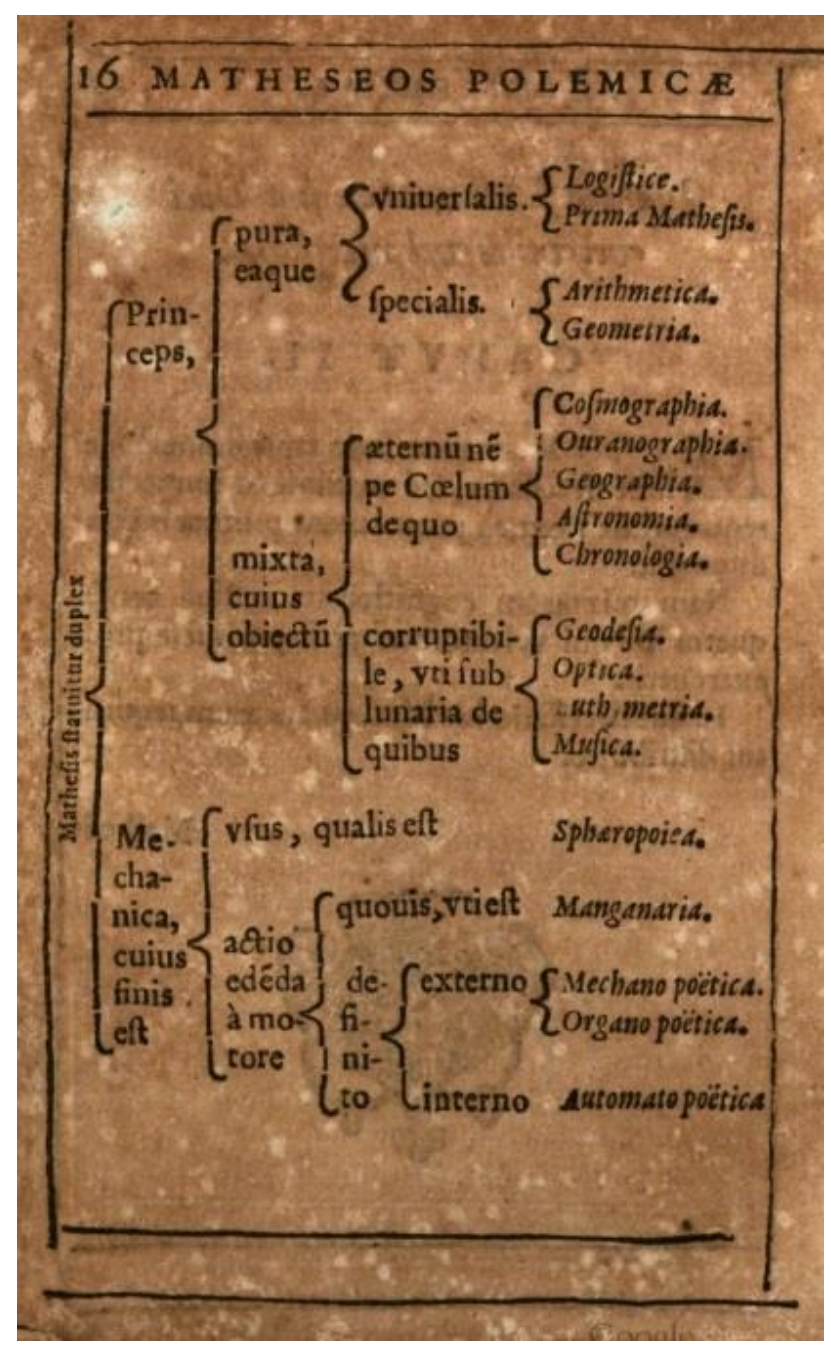

Figura 1.

Entre las principales, ocurre la división entre las matemáticas puras -aquellas que necesitan solamente del intelecto para que sean comprendidas- y las matemáticas mixtas -las disciplinas que necesitan tanto del intelecto como de los sentidos- (van Roomen 1602, pp. 14-15).

Las matemáticas puras son divididas en dos subgrupos: uno en el que están las disciplinas más generales -la prima mathesis (nombre adoptado por van Roomen para la mathesis universalis) y la logística- que sirven como base para las demás disciplinas matemáticas. Las puras especiales aritmética y geometría- que son también importantes para las demás disciplinas, pero están abajo de las universales (van Roomen 1602, pp. 14-15).

Las matemáticas puras también son divididas en dos grupos según criterios filosóficos de la materia que constituye el universo. En el primer grupo están las disciplinas que estudian los objetos celestes, como la astronomía, la astrología, la cosmografía, la uranografía y la geografía. El segundo grupo tiene como objeto los cuerpos terrenos y corruptibles, como la música, la óptica y la geodesia. Esta distinción es antigua y fue bastante difundida por Aristóteles y sus seguidores: ellos creían que el universo era dividido en dos partes: el mundo terrestre, compuesto por los cuatro elementos -aire, tierra, fuego y agua- que eran corruptibles y los objetos celestes que eran considerados eternos e incorruptibles y, por eso, compuestos por un quinto elemento (van Roomen 1602, pp. 15).

Las matemáticas mecánicas de la clasificación de van Roomen poseían nombres inventados que habitualmente no encontramos en otras clasificaciones del período. Las mecánicas son disciplinas matemáticas relacionadas a la producción, al uso y a las acciones de los instrumentos mecánicos, como los compases, los astrolabios, la palanca y otros instrumentos que eran utilizados por las disciplinas 
matemáticas o por otras disciplinas que a las matemáticas tenían gran importancia (van Roomen 1602, pp. 15-16).

La clasificación de van Roomen también considera algunas disciplinas como "casi matemáticas". Eso porque tales ciencias no eran comúnmente incluidas en las clasificaciones de otros autores, pero, según van Roomen, podrían seguramente ser consideradas matemáticas. Él ejemplifica con la arquitectura y la actividad bélica (van Roomen 1602, 1605).

\section{Obras de astronomía de los siglos XVI y XVII}

Antes de que nos fijemos específicamente en cómo van Roomen ha definido la disciplina llamada uranografía, pretendemos demonstrar que sucedió un cambio en el tipo de obra astronómica publicada en el siglo XVII respecto a las que fueron editadas en el siglo anterior. Recordamos que "a pesar de haber aumentado significativamente el número de obras de carácter técnico y científico durante el siglo XVI, ellas fueron siempre una fracción reducida del total de obras impresas" (Leitão 2004, p. 19).

Con algunas excepciones, podemos agrupar las obras astronómicas del siglo XVI principalmente en dos grupos:

- Obras filosóficas que se ocupan de la composición de los cuerpos celestes, de cómo la forma y la materia celeste están organizadas, de la causa de los movimientos celestes, de las cualidades y accidentes que los cuerpos celestes producen y su consecuente interferencia en el mundo sublunar. Muchas de estas obras están basadas en las teorías filosóficas antiguas y medievales. Podemos citar: De motibus corporum coelestium (1540) de Joannes Baptista Amici, De coelo et elementis liber (1591) de Giovanni Battista Benedetto, In libro de inteligentijs y In libro de orbibus en Opera omnia in unum collecta (1545) Alexandri Achillini y los comentarios al De caelo (1996) de Aristóteles como el In libros Aristotelis de coelo et mundo (1552) de Joannes de Janduco y el In IIII. libros Aristotelis de caelo, $\mathfrak{G}$ mundo, commentarii (1563) de Lucilio Filateo.

- Obras técnicas sobre los cuerpos celestes que se ocupan de mostrarnos la periodicidad de los movimientos celestes y cómo podemos, por ejemplo, calcular el tiempo. Esos trabajos buscan -a través de círculos y líneas imaginarias en la esfera celeste- definir reglas y fórmulas para el cálculo de la posición de los astros en determinado instante de tiempo, o también, hacer el cálculo de la posición de un individuo en la tierra. Muchas de esas obras también son dedicadas a explicaciones de la construcción y uso de instrumentos matemáticos y astronómicos. Podemos citar: Cosmographicus liber (1524) y Astronomicum caesareum (1540) de Petrus Apianus, Cosmographia in quatuor libros distributa (1598) de Francesco Barozzi, Uranometria (1603) de Johann Bayer y la gran cantidad de comentarios y traducciones al Tratado de la esfera de Sacrobosco (1472), como el In sphaeram Ioannis de Sacro Bosco commentarius (1570) de Christoph Clavius y el Tratado da sphera com a theorica do Sol e da Lua (1537) de Pedro Nunes.

Aunque estén íntimamente conectadas a la astronomía, no están en esos dos grupos las obras de astrología, pero seguramente podrían formar un tercer grupo ${ }^{1}$. Para aclarar la división en los grupos previamente enunciados, siguen algunos ejemplos de los dos tipos de obras astronómicas.

En el siglo XVII surgen obras astronómicas que unen los fundamentos filosóficos a fin de describir los problemas técnicos y matemáticos del período. Estas "nuevas" obras astronómicas surgieron como consecuencia de los debates sobre las observaciones de los cometas y de las "nuevas estrellas" que cambiaron la concepción astronómica del fin del XVI. Uno de los ejemplos más claros es la Collecta

\footnotetext{
${ }^{1}$ La astrología y la astronomía estaban íntimamente conectadas, pues el conocimiento astrológico practicado en el siglo XVI buscaba comprender cómo los cuerpos celestes podían influir en los cuerpos terrenos. No se trataba solamente de describir predicciones para el futuro, sino que comprender cómo ocurría esa influencia celeste en las cosas terrenas. Por lo tanto, la astrología tenía una relación cercana y compleja con la astronomía, la filosofía y la teología.
} 
astronomica (1603) de Christóforo Borri que presenta un modelo con una división tripartida de los cielos. En la misma línea de raciocinio, citamos la obra Sphaera triplex artificialis, elementaris, ac caelestis (1662) de Gabriele Beati.

En el siglo XVII, muchos matemáticos siguieron intentando conciliar las observaciones astronómicas y la filosofía antigua. El gran dilema fue "salvar las apariencias", o sea, llevar en cuenta los descubrimientos hechos en finales del siglo XVI y durante el XVII sin romper con la filosofía y la teología.

Aunque sea posible encontrar obras fuera de esos patrones, de modo general, podemos considerar que comúnmente las obras astronómicas producidas en el siglo XVI están divididas en los dos grupos comentados más arriba y son diferentes de las publicadas en el siglo siguiente que intentan unir los conocimientos técnico-matemáticos y filosóficos. En la próxima sección mostraremos justamente cómo la obra Ouranographia de van Roomen publicada en el final del siglo XVI escapa de los debates de aquel momento.

\section{La astronomía y la uranografía}

Van Roomen escribió tres trabajos de astronomía. El Speculum astronomicum siue organum forma mappae expressum (1606) describe técnicamente los círculos del primer cielo y del primer móvil mostrando, por ejemplo, métodos, reglas y tablas de cálculos astronómicos con el uso de triángulos esféricos. Claramente esta obra aborda solamente cuestiones técnicas de la astronomía y podría estar incluida en el segundo grupo de obras a pesar de haber sido publicada en el inicio del siglo XVII.

La segunda, en realidad, es la tesis cuyo título es Theses astronomicae quibus proponuntur nonnulla de corporum mundanorum simplicium disctinctione et número (1598) y fue producida por Lambertus Croppet, discípulo de van Roomen. Ese trabajo de 1598 es compuesto por dos partes: la primera describe los elementos constituyentes de los cuerpos celestes y terrestres, la diferencia entre objetos sensibles e inteligibles en el mundo supralunar y las diferencias entre estrellas fijas y erráticas. La segunda parte es un conjunto de tablas de cuerdas, pero sin ningún contenido. Sin embargo, no podemos decir que esa obra debería ser considerada como tratado filosófico y matemático a la vez, pues su segunda parte no es presentada como una conexión con los asuntos abordados en la primera.

La tercera obra, de la que hablaremos más adelante, la Ouranographia sive caeli descriptio o Uranografía o la descripción del cielo (1591), publicada en 1591 diferencia la uranografía de la astronomía y también nos muestra como la primera puede ser una conexión entre matemáticas y filosofía.

Normalmente la astronomía está presente en las diversas clasificaciones de las matemáticas publicadas en los siglos XVI y XVII. Esa ciencia, según el propio van Roomen, poseía una parte principal denominada uranografía. En el capítulo siete de la obra Universae mathesis idea (1602), él distingue la astronomía de la uranografía de la siguiente manera: "La astronomía es la ciencia del movimiento de los cuerpos celestes. La uranografía distingue el cielo en sus partes sensibles e inteligibles. Sensibles son las estrellas y la vía láctea, pero inteligibles son las esferas y los círculos. Y además la uranografía es una parte de la astronomía y, seguramente, la primera" (van Roomen 1602, p. 26).

La palabra latina uranus deriva del término griego Ov̉ oxvós que en la mitología significa Urano, padre de Saturno. Sin embargo, también puede señalar el cielo o la personificación de los cielos. De esa manera, la palabra uranografía muestra una construcción de la representación celeste o una descripción del cielo, como indica el título de la obra de van Roomen.

Según van Roomen, el objeto de estudio de la astronomía es el cielo y sus movimientos. Por otro lado, la uranografía tiene otro enfoque: entender las partes de los cielos según las definiciones aristotélicas de cuerpos sensibles e inteligibles. Según la teoría aristotélica, sensibles son las cosas que pueden ser percibidas por los sentidos. En ese caso, sensibles son los planetas y las estrellas, pues pueden ser mirados por los ojos humanos. Por otro lado, inteligibles son las cosas que solamente pueden ser conocidas a través del intelecto y, en la uranografía, van Roomen se refiere a los círculos y 
líneas imaginarias que los astrónomos utilizaban para hacer los cálculos astronómicos y para que se ubicaran en el mar o en la tierra. Como van Roomen también tenía ganas de demonstrar la importancia de la astronomía en las actividades bélicas, él nos dice que la astronomía es importante para el soldado en la guerra, principalmente cuando está en el mar, para que tenga conocimiento suficiente para examinar su localización (van Roomen 1602, pp. 26, 32).

Van Roomen también dice que a veces el cielo debe ser comprendido como un cuerpo continuo y en otras como contiguo. Continuo pues es compuesto por un único elemento de modo que algunas partes se quedan más espesas (las estrellas y planetas visibles) y en otras más raras (los orbes celestes que cargan los planetas y estrellas) que no podemos verlas justamente debido a esa pequeña densidad. La vía láctea es un ejemplo dado por el autor de una región celeste de densidad mediana (van Roomen 1602).

A través de las esferas y círculos creados por la uranografía está permitido describir los sitios de los astros en cualquier instante de tiempo, aunque el astrónomo esté en algún lugar donde no se pueda ver el cielo, como una cárcel oscura. Sin embargo, las observaciones no pueden ser descartadas, porque son ellas que permiten establecer las reglas astronómicas y confirmar las hipótesis (van Roomen 1602, pp. 26-27).

La finalidad de la astronomía es definir el movimiento de todos los astros, y después sus posiciones en cualquier instante de tiempo. Esa disciplina tiene como principio el conocimiento de la geometría y de la aritmética: los principios geométricos sirven para crear las reglas de los movimientos de los cuerpos celestes y los principios aritméticos sirven para realizar el cálculo de tales reglas. Las principales propiedades de la astronomía son buscar la longitud, la latitud, el ascenso y descenso de los astros, la declinación, la altitud, la distancia, la conjunción y la oposición de los astros (van Roomen 1602, p. 27).

Además, entre las matemáticas mixtas, van Roomen define que la astronomía obtiene el primer lugar debido a la naturaleza noble de los objetos celestes, considerando los principios aristotélicos de que los cielos son compuestos por el quinto elemento diferente de los cuatro elementos terrestres aire, fuego, tierra y agua. Ese quinto elemento es diferente de los cuatro elementos que forman las cosas terrenas porque el movimiento celeste circular que no cambia de velocidad y de dirección, es considerado incorruptible y eterno. A los cuatro elementos existentes en el mundo terrestre es posible haber corrupción y generación (van Roomen 1602, p. 27).

El autor también nos muestra que la astronomía, así como otras ciencias, no fue creada o cultivada en un único tiempo, pero fue el resultado de muchas generaciones de estudio. Para eso, van Roomen cita fragmentos de la biblia y de trabajos de la antigüedad y de la edad media (van Roomen 1602, pp. 29-32).

La astronomía fue dividida por Roomen en tres partes: (i) la astronomía lógica que muestra las formas y datos para raciocinar. El autor parece referirse a los datos que pueden ser retirados de la observación de los movimientos celestes y posteriormente analizados; (ii) la astronomía meteoroscópica que es responsable por las observaciones con el uso de instrumentos científicos y; (iii) la astronomía metódica que muestra la estructura de toda la ciencia astronomía, o sea, van Roomen se refiere a las reglas, a los principios básicos y a los modos de raciocinar en los estudios y pesquisas astronómicas (van Roomen 1602, p. 32).

\subsection{Ouranographia sive caeli descriptio}

La Ouranografia sive caeli descriptio (1591) es una obra muy importante para la historia de la ciencia y de la astronomía, pues nos muestra una disciplina diferente de la astronomía. La uranografía es una parte importante de la astronomía que intenta unir los conocimientos de las matemáticas a la filosofía.

Esa fue la primera obra publicada por van Roomen, en 1591, y actualmente encontramos dos frontispicios diferentes (figura 2): el ejemplar de la Bayerische Staatsbibliothek (Alemania) fue imprimido por Joahnnes Keerbergium en Antwerpen; por otro lado, la copia de la 
Universiteitsbibliotheek Gent (Bélgica) fue producida en el mismo año por Johannes Masi en Leuven (van Roomen 1591).

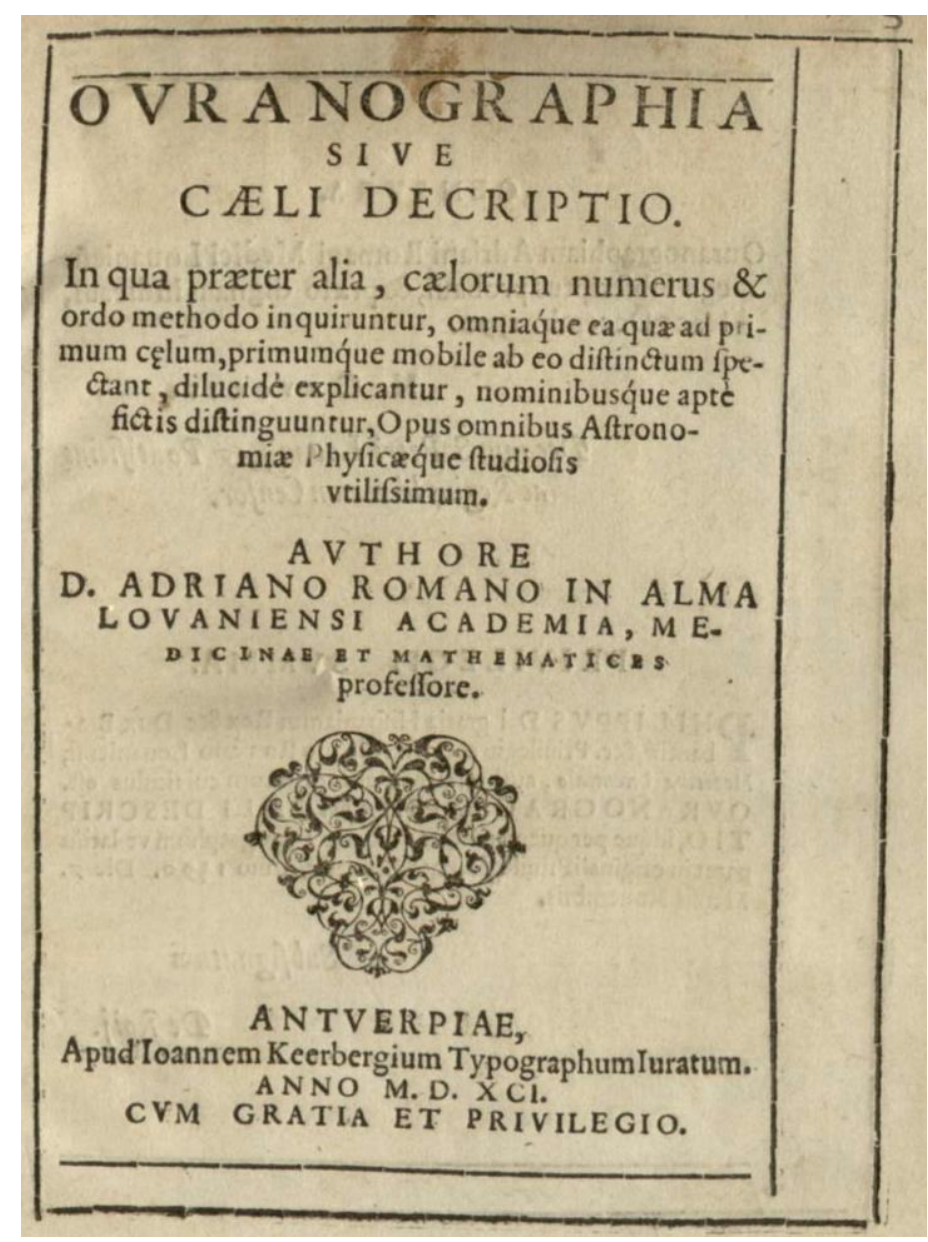

Figura 2.

La obra está dividida en una parte introductoria y tres libros: el liber primus proporciona una descripción de la "máquina celeste como un todo", la segunda y tercera partes, abordan los círculos y movimientos del primer cielo y del primer móvil, respectivamente (van Roomen 1591).

La parte introductoria está compuesta por el frontispicio; la censura y el resumen del privilegio (extractos de los documentos que contienen la autorización del Rey para la publicación de la obra); por una carta y dedicatoria a António Heetveldio, alcalde de Leuven; el prefacio y; la "división general de nuestra Ouarnographia", un esquema con un tipo de sumario del contenido de la obra.

El liber primus tiene veintidós capítulos en los cuales van Roomen explica filosóficamente la composición, organización y movimiento de los cuerpos celestes. Como hemos dicho anteriormente, para el autor, la estructura general de los cuerpos celestes puede ser explicada de dos modos. De una primera manera, él explica que los cielos pueden ser considerados un cuerpo único, describiendo cuál sería su esencia y sus accidentes. La esencia habla de la materia y forma de los cielos. Los términos son derivados de la filosofía aristotélica: "la materia es el sujeto primero de una cosa, por eso, la cosa no puede ser generada accidentalmente; es lo que permanece a través de los cambios"; forma "no se opone a la materia, pero la presupone". La forma es la "esencia o substancia de las cosas que tienen materia; es causa o razón de ser de la cosa; que está en virtud de lo que una cosa es lo que es" (Abbagnano 2007, p. 646). Durante los primeros capítulos, van Roomen diferencia algunos de los puntos de la filosofía aristotélica y platónica. Para él, citando obras de Aristóteles, la materia del cielo es el quinto elemento y la forma es perfecta e incorruptible debido a la propia naturaleza de la materia que lo compone (van Roomen 1591, pp. 5-7). 
El autor presenta también las cualidades sensibles existentes en los cielos: la luz que las estrellas y planetas emiten y la cualidad táctil que habla sobre la densidad del quinto elemento en las distintas regiones celestes: existen regiones en las cuales los cuerpos celestes son más raros y otras en las que son más espesos. La densidad de las regiones celestes es justificación para van Roomen sobre el hecho de que los cielos son divisibles en partes y, al mismo tiempo, porque son compuestos por un único elemento, es un cuerpo continuo (van Roomen 1591, p. 8).

Sobre las acciones de los cielos, van Roomen se remite a los movimientos ejercidos por las esferas celestes. Debido a la naturaleza del quinto elemento, "para consolidar su perfección" y "para asimilarse a Dios", ese movimiento es siempre circular y perpetuo, nunca cambia de dirección o velocidad (van Roomen 1591, p. 9).

El autor también explica que los cielos pueden ser tratados como un cuerpo contiguo, o sea, divisible en distintas esferas celestes, pero cada una de esas partes se tocan y causan influencias en las demás esferas. Para eso, van Roomen describe cuál es el orden y el número de las esferas celestes según las opiniones de diversos expertos, como astrónomos, poetas y teólogos, a lo largo de la historia (van Roomen 1591, pp. 13-20).

Van Roomen describe el orden de los orbes, de arriba hacia abajo, de las siguientes maneras: primero el primer cielo, después el primer móvil, el cristalino, el firmamento o el cielo de las estrellas fijas, Saturno, Júpiter, Marte, Sol, Venus, Mercurio, y, por último, la Luna que está cerca de la Tierra. Las esferas del firmamento y del cristalino fueron incluidas en el sistema del mundo a fin de explicar otros movimientos de los astros, como el actualmente llamado precesión de los equinoccios (Van Roomen 1591, pp. 19-20).

El no muestra ninguna figura del sistema del mundo que adopta en su Ouranographia, pero podemos usar como ejemplo la figura del Cosmosgraphicus liber (1524) de Apianus (figura 3).

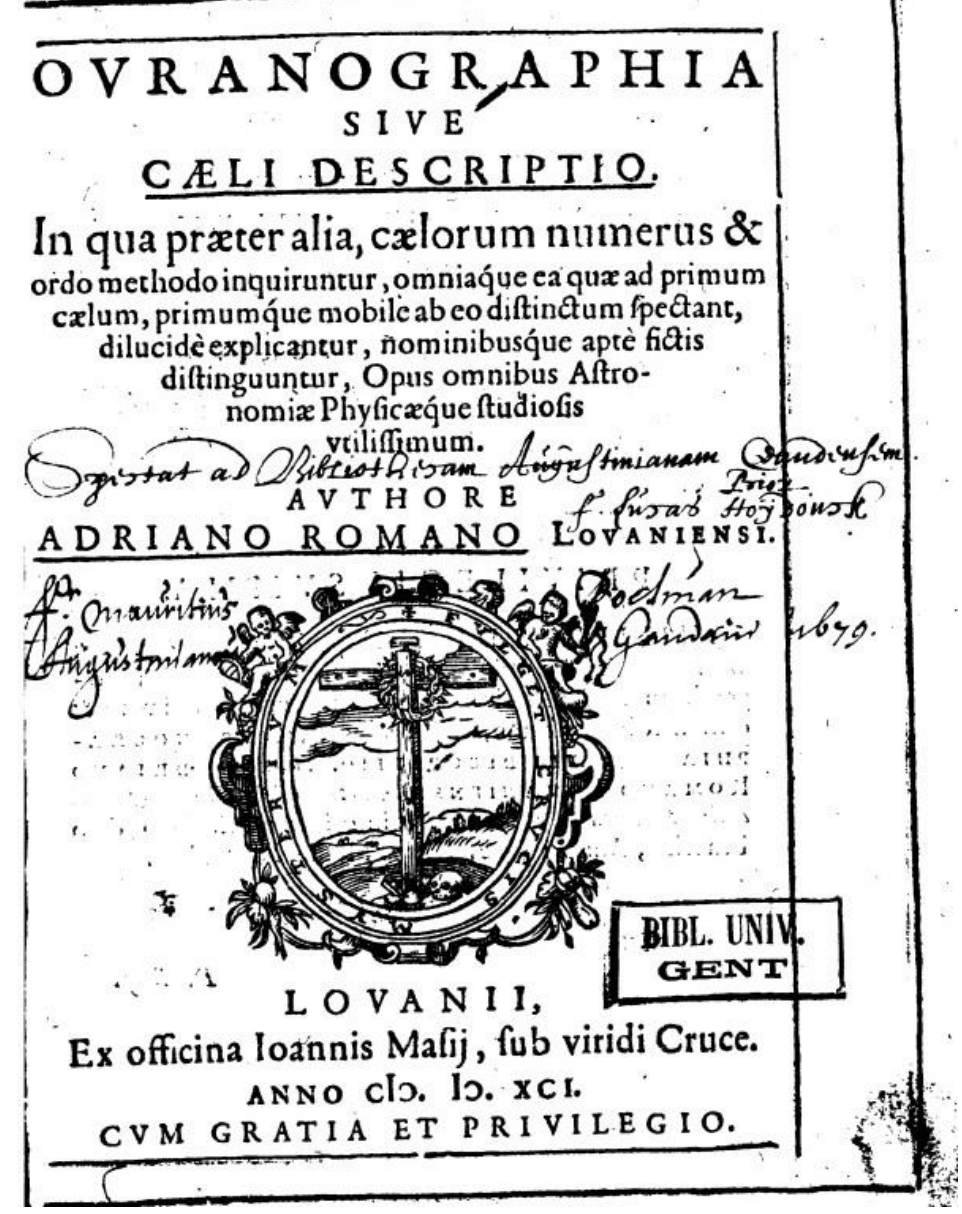

Figura 3. 
Ese enfoque duplo - los cielos como cuerpos continuos y contiguos- es dado por el autor para que tengamos diferentes puntos de vista de la "máquina celeste", abordando el máximo de aspectos filosóficos conectados a la astronomía.

Ese primer libro es basado principalmente en obras de Aristóteles y de seguidores de la filosofía peripatética, como Averróis y Tomás de Aquino. Además, van Roomen también cita diversos escritos astronómicos, poéticos, filosóficos y teológicos de autores de la antigüedad, de la Edad Media y del Renacimiento.

En la figura 4, hicimos un esquema para demonstrar las ideas descritas en el liber primus. Aclaramos la bifurcación entre el cielo considerado único y divisible y las respectivas características importantes debatidas por el autor. ${ }^{2}$

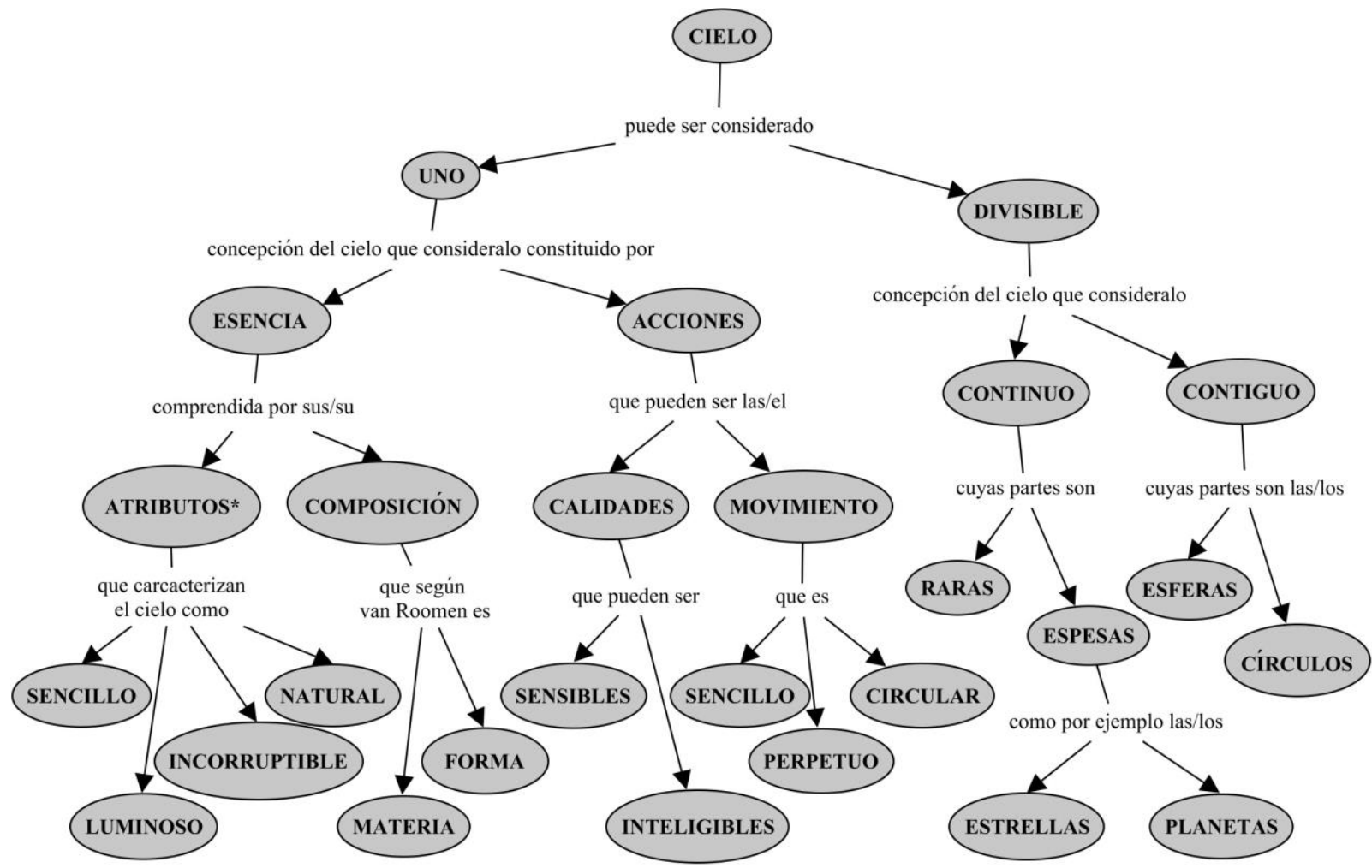

Figura 4.

Como hemos dicho anteriormente, en el liber secundus y tertius el enfoque cambia: van Roomen ya no se dedica exclusivamente a las ideas filosóficas (aunque no las abandona), pero pasa a describir los círculos y movimientos del primer cielo y del primer móvil.

El primer cielo (primum caelum) es la esfera más externa de todas, inmóvil, que los "cristianos lo denominan empíreo, morada de Dios, de los ángeles y de los santos”. Algunos de los círculos imaginarios de esa esfera son: el ecuador y sus paralelos, el horizonte, la vertical y el meridiano (van Roomen 1591, pp. 28-29).

El primer móvil (primum mobile) es la esfera que viene en seguida del primer cielo y, como el propio nombre dice, es responsable por el primer movimiento, el movimiento diurno, o sea, el movimiento del que nos damos cuenta a través de la variación de los días y de las noches. Algunos de sus círculos son el mediador, la eclíptica, el zodiaco y sus signos, y algunos paralelos importantes como el trópico de Cáncer, el trópico de Capricornio, el círculo ártico y el círculo antártico (van Roomen 1591, p. 45).

A pesar del cambio de enfoque de los segundos y terceros libros, van Roomen no abandona las concepciones abordadas en el liber primus presentando los círculos celestes, su tecnicidad y sus usos siempre con respecto a los conceptos filosóficos y teológicos. Por ejemplo, el mediador, un círculo que divide el primer móvil en dos partes iguales es llamado así porque es a través de él que las influencias

\footnotetext{
2 Adoptamos el término "atributos", aunque van Roomen no hace uso de esa palabra en su trabajo.
} 
del primer cielo son transmitidas a las esferas inferiores y a la Tierra. En el caso del zodiaco, el autor muestra que esta es la causa de la existencia de vida en la Tierra y también sirve como signo o marca, porque cuando es cubierto por el Sol, marca el tiempo y muestra el hogar de los planetas, además, su oblicuidad causa diferencia de duración de los días a lo largo del año. Así, el autor muestra que los círculos no sirven solamente como marcadores, pero también tienen su importancia filosófica (van Roomen 1591).

\section{La uranografía y las relaciones de las disciplinas matemáticas con la filosofía}

La diferencia de la Ouranographia sive caeli description (1591) con respecto a las obras del siglo XVI es notable, pues van Roomen no se dedica solamente a los aspectos filosóficos o solamente a los aspectos técnico-matemáticos de la astronomía. En esa obra, él claramente une los dos temas, fundamentando inicialmente la astronomía a partir de los preceptos de la filosofía -principalmente la aristotélica- y, después, empieza a escribir acerca del punto de vista matemático, pero nunca abandona los debates filosóficos.

Sin embargo, la Ouranographia tampoco puede ser colocada como precursora de las obras astronómicas - producidas por los jesuitas o no- del siglo XVII, pues ella tiene como idea central el geocentrismo. Diferentemente, la Collecta astronomica (1631) de Borri, por ejemplo, busca discutir una "nueva" astronomía a partir de una organización celeste distinta del geocentrismo ptolemaico y aristotélico. Esa obra intenta mostrar que los cielos son fluidos y su división es tripartida: los cielos aéreo, sidéreo y empíreo (Borri 1631, pp. 257-267). Otra obra que muestra la misma idea es la Sphaera triplex artificialis, elementaris, ac caelestis (1662) de Beati, que como el título dice, también divide los cielos en tres (pp. 1, 53-55, 103).

Aunque la Ouranographia sea una obra que lleva en cuenta el sistema de mundo aristotélicoptolemaico, de manera alguna podemos decir que van Roomen estaba inconsciente de los "nuevos" sistemas de mundo que eran estudiados en aquel tiempo. Por el contrario, sabemos a través de su correspondencia, que el autor tuvo frecuencia asidua en las ferias de libros de Mainz y de Frankfurt y siempre buscó leer y adquirir las obras científicas que estaban siendo publicadas, además su círculo de amistades era compuesto de grandes nombres de la ciencia de aquel tiempo. El impacto de esa cuantidad de lecturas puede ser visto por sus citaciones en la Ouranographia y en otras de sus obras. Recordemos también que entre los años 1599 y 1601 -o sea, menos de una década después de la publicación de la Ouranographia- van Roomen tuvo la oportunidad de visitar a Johannes Kepler y Tycho Brahe.

No obstante, esos factos no son suficientes, pues no sabemos cuándo han empezado esas amistades y cuándo van Roomen tuvo contacto con las teorías astronómicas de esos científicos por primera vez. Pero, el facto de que en un fragmento de la Ouranographia van Roomen mencione la teoría heliocéntrica de la De revolutionibus (Copérnico 1543) y el poema heliocéntrico de Cornelius Gemma (Hallyn 2008), demuestra que él conocía los debates acerca del heliocentrismo. Sin embargo, él parece no valorar a esos puntos de interés de la astronomía de su período. En cualquier de los dos casos citados, él no se posiciona ni nos dice su opinión respecto a ese sistema de mundo, lo que nos da la impresión de haber ignorado tales debates. No obstante, es importante notar que van Roomen tenía conocimiento de la existencia de esos "nuevos" sistemas de mundo, pero prefirió escribir la Ouranographia completamente basada en la filosofía aristotélico-ptolemaica, dejando de lado los debates con respecto al sistema heliocéntrico que habían empezado casi medio siglo antes.

En la citación que hicimos en la Introducción, Capel (1989) nos muestra que las omisiones y silencios tienen gran significado para nosotros historiadores. Resaltamos que van Roomen no fue el único a aparentemente ignorar el sistema de mundo heliocéntrico. En realidad, pocos estudiosos publicaron trabajos sobre el sistema copernicano en el siglo XVI. Henrique Leitão (2002, pp. 60-61) afirma que en el siglo XVI la tesis heliocéntrica elaborada por Copérnico no fue una de las cuestiones agudas de aquel momento. Robert S. Westman (1980, pp. 136) nos muestra los pocos nombres que 
adhirieron a la concepción copernicana afirmando que "entre 1543 y 1600, yo [Westman] encontré no más que diez pensadores que decidieron optar por la pretensión principal de la teoría heliocéntrica” y explicita los siguientes nombres: "Thomas Digges y Thomas Harriot en Inglaterra; Giordano Bruno y Galileo Galilei en Italia; Diego de Zúñiga en España; Simon Stevin en los Países Bajos; y, en Alemania, -el mayor grupo- Georg Joachim Rheticus, Michael Maestlin, Christopher Rothmann y Johannes Kepler”. Leitão (2002, pp. 61) afirma que para que esa lista esté correcta, debemos incluir el nombre de Reiner Gemma Frisius y retirar el de Rothmann, porque, aunque inicialmente haya adoptado las teorías heliocéntricas, después de conocer a Kepler, las abandonó. ${ }^{3}$

Consideramos que la uranografía de van Roomen, además de ser una disciplina matemática con concepciones de astronomía de la antigüedad y de la edad media, es una "nueva" disciplina debido a su manera de abordar las relaciones entre conocimiento filosófico y mecánico (técnico-matemático). Ella hace parte de las matemáticas mixtas y, buscando fundamentar filosóficamente la tecnicidad de la astronomía geocéntrica, es también parte importante y principal de la ciencia astronómica.

Creemos que esa relación entre matemáticas y filosofía hecha por van Roomen, directa o indirectamente, está conectada al debate acerca de la certeza de las matemáticas, porque, para que los matemáticos alcanzaran un estatuto mejor para las matemáticas en los colegios jesuitas y en las universidades, uno de los caminos sería introducir conceptos y conocimientos filosóficos para fundamentar y valorar las matemáticas. Van Roomen, de alguna manera, se une a los matemáticos que intentaban mostrar a los filósofos que las matemáticas poseían conocimiento cierto no solamente por su manera de demostrar, pero también porque podían fundamentar el conocimiento matemático en las concepciones aristotélicas que eran bien aceptadas en aquel tiempo. Aunque el autor no mencione en ningún momento la quaestio de certitudine mathematicarum, las características diferenciadas de la obra de van Roomen y sus cartas enviadas a Clavius demuestran que él seguramente ansiaba que las disciplinas matemáticas alcanzaran un mejor status, al menos en las universidades y en las regiones que estaba trabajando. Su interés posterior en describir y teorizar acerca de la mathesis universalis nos muestra que él realmente quería valorar el conocimiento matemático frente a las demás ciencias (van Roomen 1597).

Sin duda, ese trabajo no es definitivo, pues las posiciones adoptadas por van Roomen no pueden ser generalizadas para todo el continente europeo. Características específicas de cada región y de cada período influyen de manera diversa en el desarrollo y en la práctica de las matemáticas.

La elección del autor en la obra Mathesis polemica (1605) por las actividades de guerra pueden estar justamente conectadas debido a los conflictos que ocurrieron en su región o -como afirma Mario Biagioli (1989) en el caso específico de Italia, pero que puede eventualmente ser generalizado para otros lugares- porque estaba ocurriendo una creciente profesionalización de las actividades militares y un perfeccionamiento de los instrumentos bélicos. Por otro lado, en la península ibérica, por ejemplo, el interés por las navegaciones hacía parte de los debates centrales, lo que futuramente propiciaría la escrita de muchos trabajos de astronomía con el intuito de perfeccionar las navegaciones (Navarro Brotóns 2000).

Sabemos que lo que hemos presentado es solamente una pequeña parte de la historia de la matemática en finales del siglo XVI, y creemos que la Ouranographia de van Roomen nos ayuda a comprender -a través del punto de vista del propio autor- algunos debates más generales acerca de las disciplinas matemáticas. Es posible percibir a través de esa obra y de sus cartas que el estatuto de las matemáticas afectaba directamente sus ocupaciones profesionales y que él siempre tenía poco tiempo para dedicarse a los temas matemáticos de su interés. Aunque no haya habido consenso entre los expertos y científicos de los siglos XVI y XVII, el texto de van Roomen nos muestra algunas características de cómo estaban clasificadas y organizadas las disciplinas matemáticas y cómo los debates sobre la certeza del conocimiento matemático y la idea de mathesis universalis también influían en el desarrollo de las matemáticas.

\footnotetext{
${ }^{3}$ Seguramente es necesario que intentemos profundizar las afirmaciones de Westmann (1980) y Leitão (2002), pero nuestro interés central es mostrarle al lector que el copernicanismo no fue una de las principales discusiones en el siglo XVI.
} 
Bibliografía

Achillini, A. (1545), Opera omnia in unum collecta, Venezia: Hieronymum Scotum.

Amici, J. B. (1540), De motibus corporum coelestium juxta principia peripapetica, Paris: Jacob Kerver.

Apianus, P. (1524), Cosmographicus liber, Landshut: Joannis Weyssenburgers.

Apianus, P. (1540), Astronomicum caesareum, Ingolstadt: Georg y Petrus Apianus.

Aristóteles (1996), Acerca del cielo, Madrid: Gredos.

Barozzi, F. (1598), Cosmographia in quatuor libros distributa, Venezia: Gracioso Perchacino.

Bayer, J. (1603), Uranometria, Augsburg: Mangus Christophorus.

Beati, G. (1662), Sphaera triplex artificialis, elementaris, ac caelestis, Roma: Typis Varesij.

Benedetto, G. (1591), De coelo et elementis liber, Ferrara: Victorius Baldinus.

Biagioli, M. (1989), “The Social Status of Italian Mathematicians, 1450-1600”, History of Science 27: 41-95.

Bockstaele, P. (1976), "The Correspondence of Adriaan van Roomen", LIAS - Sources and Documents relating to the Early Modern History of Ideas 3: 85-129, 249-299.

Bockstaele, P. (1992), "The Correspondence of Adriaan van Roomen (1561-1615): Corrections and Additions, 1594 1615”, LIAS - Sources and Documents relating to the Early Mordern History of Ideas 19: 3-20.

Bockstaele, P. (2009), "Between Viète and Descartes: Adriaan van Roomen and the Mathesis Universalis”, Archive for History of Exact Sciences 63(4): 433-470.

Borri, C. (1631), Collecta astronômica, Lisboa: Mathias Rodrigues.

Busard, H. L. L. (1981), "Roomen, Adriaan van”, en Gillispie, C. C. (ed.). Dictionary of Scientific Biography, vol. 11, New York: Scribner, pp. 532-534.

Capel, H. (1989), "Historia de la Ciencia e Historia de las Disciplinas Científicas”, GeoCrítica: Cuadernos Críticos de Geografia Humana XII(84). http://www.ub.edu/geocrit/geo84c.htm Web (acceso: 15 de mayo de 2017).

Carolino, L. M. (2006), “João Delgado SJ e a ‘Quaestio de Certitudine Mathematicarum’ em Inícios do Século XVII”, Revista Brasileira de História da Matemática 6(11): 17-49.

Carolino, L.M. (2009), "Cristoforo Borri e o Impacto da Nova Astronomia em Portugal no Século XVII", Revista Brasileira de História da Ciência 2(2): 160-181.

Clavius, C. (1570), In sphaeram Ioannis de Sacro Bosco commentaries, Roma: Victorium Helianum.

Copérnico, N. (1543), De revolutionibus orbium coelestium, Norimbergae: J. Petreium.

Crapulli, G. (1969), Mathesis Universalis: genesi di una'idea nel XVI secolo, Roma: Edizioni dell'Ateneo.

Descartes, R. (1684), Regulae ad directionem ingenii, Amsterdam: Jan Rieuwertsz.

Euclides (2009), Os elementos, São Paulo: UNESP.

Filateo, L. (1563), In IIII. libros Aristotelis de caelo, et mundo, commentarii, Venezia: V. Valgrisium.

Gonçalves, C. H. B. y Z. V. Oliveira (2010), “A Atividade Matemática de Adriaan van Roomen”, Revista Brasileira de História da Matemática 10(20): 151-168.

Hallyn, F. (2008). "A Poem on the Copernican System: Cornelius Gemma and His Cosmocritical Art”, en Hirai, H. (ed.), Cornelius Gemma: Cosmology, Medicine and Natural Philosophy in Renaissance Louvain, Pisa: Fabrizio Serra, pp. $13-32$.

Janduco, J. de (1552), In libros Aristotelis de coelo et mundo, Venezia: apud Juntas.

Knobloch, E. (1989), “Klassifikationen”, en Folkerts, M. (ed.), Mass, Zahl und Gewicht - Mathematik als Schlüssel zu Weltverständnis und Weltbeherrschung, Weinheim: VCH, Acta Humaniora, pp. 13-40.

Leitão, H. (2002), “Uma Nota sobre Pedro Nunes y Copérnico”, Gazeta de Matemática 143: 60-78. 
Leitão, H. (2004), "O Livro Científico Antigo, Séculos XV e XVI: notas sobre a situação portuguesa”, en Leitão, H. (ed.), O Livro Científico Antigo dos séculos XV e XVI. Ciências físico-matemáticas na Biblioteca Nacional. Catálogo de livros científicos dos séculos XV e XVI, Lisboa: Ministério da Cultura e Biblioteca Nacional de Portugal, pp. 15-53.

Mancosu, P. (1996), Philosophy of Mathematics and Mathematical Practice in Seventeenth Century, New York: Oxford University Press.

Navarro Brotóns, V. (2000), "Astronomía y cosmografía entre 1561 y 1625: aspectos de la actividad de los matemáticos y cosmógrafos españoles y portugueses", Cronos: Cuadernos valencianos de historia de la medicina y de la ciencia 3(2): 349-380.

Oliveira, Z.V. (2015), A Classificação das Disciplinas Matemáticas e a Mathesis Universalis nos Séculos XVI e XVII: um estudo do pensamento de Adriaan van Roomen, Tesis Doctoral, Universidade Estadual Paulista "Júlio de Mesquita Filho".

Sasaki, C. (2004), Descartes's Mathematica Thought, New York: Springer.

Sacrobosco, J. (1472), Tractatum de Spera, Venezia: Florentius de Argentina.

van Roomen, A. (1591), Ouranographia sive caeli description, Antwerpen: Johannes van Keerbergen. ${ }^{4}$

van Roomen, A. (1593), Ideae mathematicae pars prima, Antwerpen: Johannes van Keerbergen. ${ }^{5}$

van Roomen, A. (1597), In Archimedis circuli dimensionem expositio et analysis, Würzburg: Georg Fleischmann.

van Roomen, A. (1598), Theses astronomicae quibus proponuntur nonnulla de corporum mundanorum simplicium disctinctione et numero, Würzburg: Georgius Fleischmann.

van Roomen, A. (1602), Universae mathesis idea, Würzburg: Georgius Fleischmann.

van Roomen, A. (1605), Mathesis polemica, Frankfurt: Levinus Hulsius.

van Roomen, A. (1606), Speculum astronomicum siue organum forma mappae expressum, Leuven: Johannes Masi.

Weber, J.P. (1964), "Sur la Composition de la Regula IV de Descartes", Revue Philosophique de la France et de l'Étranger 89(154): $1-20$

Westman, R.S (1980), "The Astronomer's Role in the Sixteenth Century: A Preliminary Study", History of Science 18: 105-147.

${ }^{4}$ Algunos ejemplares aparecen como impresa en Leuven por Johannes Masi.

${ }^{5}$ Algunos ejemplares aparecen como impresa en Leuven por Johannes Masi. 